\title{
Distractor interference in selective reaching: effects of hemispace, movement direction, and type of movement
}

Citation for published version (APA):

Keulen, R. F., Adam, J. J., Fischer, M. H., Kuipers, H., \& Jolles, J. (2007). Distractor interference in selective reaching: effects of hemispace, movement direction, and type of movement. Cortex, 43(4), 531541. https://doi.org/10.1016/S0010-9452(08)70247-8

Document status and date:

Published: 01/01/2007

DOI:

10.1016/S0010-9452(08)70247-8

Document Version:

Publisher's PDF, also known as Version of record

Please check the document version of this publication:

- A submitted manuscript is the version of the article upon submission and before peer-review. There can be important differences between the submitted version and the official published version of record.

People interested in the research are advised to contact the author for the final version of the publication, or visit the DOI to the publisher's website.

- The final author version and the galley proof are versions of the publication after peer review.

- The final published version features the final layout of the paper including the volume, issue and page numbers.

Link to publication

\footnotetext{
General rights rights.

- You may freely distribute the URL identifying the publication in the public portal. please follow below link for the End User Agreement:

www.umlib.nl/taverne-license

Take down policy

If you believe that this document breaches copyright please contact us at:

repository@maastrichtuniversity.nl

providing details and we will investigate your claim.
}

Copyright and moral rights for the publications made accessible in the public portal are retained by the authors and/or other copyright owners and it is a condition of accessing publications that users recognise and abide by the legal requirements associated with these

- Users may download and print one copy of any publication from the public portal for the purpose of private study or research.

- You may not further distribute the material or use it for any profit-making activity or commercial gain

If the publication is distributed under the terms of Article 25fa of the Dutch Copyright Act, indicated by the "Taverne" license above, 


\title{
RESEARCH REPORT
}

\section{DISTRACTOR INTERFERENCE IN SELECTIVE REACHING: EFFECTS OF HEMISPACE, MOVEMENT DIRECTION, AND TYPE OF MOVEMENT}

\author{
Ron F. Keulen ${ }^{1}$, Jos J. Adam², Martin H. Fischer ${ }^{3}$, Harm Kuipers ${ }^{2}$ and Jelle Jolles ${ }^{1}$ \\ $\left({ }^{1}\right.$ Department of Psychiatry and Neuropsychology, and ${ }^{2}$ Department of Movement Sciences, \\ Maastricht University, Maastricht, The Netherlands; ${ }^{3}$ Department of Psychology, University of Dundee, \\ Dundee, Scotland, UK)
}

\begin{abstract}
In this study we investigated the influence of hemispace, movement direction, and type of movement on distractor interference in selective reaching. Participants reached for a green target while ignoring a simultaneously presented red distractor. In Experiment 1 participants performed rightward or leftward movements within the right or the left hemispace using their dominant (i.e., right) hand. Reaction times, movement times, and percentage errors were recorded. Results showed significant interference effects in movement time, not in reaction time. Importantly, movement time interference was found to be smaller for leftward than for rightward movements. However, in Experiment 1, movement direction was confounded with type of movement (i.e., abduction $v s$. adduction). In Experiment 2 we disentangled these two factors by having participants perform rightward and leftward movements with right and left hands. Results indicated again that leftward movements were less prone to distractor interference than rightward movements, regardless of the responding hand. This phenomenon is interpreted in terms of a left hemisphere superiority in online feedback-processing during goaldirected movements in right-handers.
\end{abstract}

Key words: selective reaching, distractor interference, movement direction, movement type, hemispace

\section{INTRODUCTION}

Reaching for and grasping objects in space (i.e., manual aiming) is an activity we perform many times each day, for example when pushing a button on a keyboard or grasping a pencil from the table. There is an extensive literature on manual aiming, focusing on topics such as manual asymmetries (i.e., right hand $v s$. left hand responses, preferred hand $v s$. non-preferred hand responses), differences in manual aiming performance to targets at various spatial locations, and manual aiming performance as a function of the presence or absence of taskirrelevant stimuli (i.e., distractors). In the present study we sought to draw these different literatures together through investigating the effect of distractors on manual aiming performance as a function of the responding hand and the spatial location of the target. First, we will discuss the terminology that is used when referring to aiming movements to targets at various locations, followed by a review of the literature on manual aiming performance as a function of target location and responding hand. Then we will discuss the effects of distractor objects on manual aiming performance.

In studies of manual aiming, different terms are used for labeling aiming movements toward various spatial locations. The terms ipsilateral and contralateral are used for indicating the relation between the hemispace in which the aiming response is performed and the responding hand. Ipsilateral means on the same side of space as the side of the responding effector; contralateral means on the side of space opposite to the side of the responding effector. For the direction of the required response, on some occasions the terms adduction and abduction are used, whereas in other occasions the terms rightward and leftward are used. An abduction refers to an aiming response commencing in contralateral space or at the midsagittal plane and heading for the ipsilateral side of space. An adduction, on the other hand, commences in ipsilateral space or at the midsagittal plane and heads for the contralateral side of space. Thus, both abduction and adduction responses might (but not necessarily need to) cross the body midline. The terms rightward and leftward speak for themselves. In order to prevent any confusion to occur, from now on we will use the term movement direction when differentiating between rightward and leftward movements. On the other hand, when differentiating between abductive and adductive responses, we will use the term movement type.

It should be clear from the above definitions, that the terms used to label target-directed aiming responses are easily confounded. For instance, an adductive movement that commences at the midsagittal plane takes place in contralateral space, whereas an adductive response that commences on the ipsilateral side of space and terminates at the midsagittal plane can be qualified as a movement in ipsilateral space. Furthermore, when moving with the right hand, an adduction is always directed to the left and an abduction to the right, whereas the opposite is true when moving with the 
left hand. In the present study we sought to unconfound the effects of hemispace, movement direction, and type of movement.

Manual aiming performance as a function of the spatial location of the target has been studied both in multi-element aiming movements toward multiple targets and in discrete aiming movements toward a single target. Regarding the studies into continuous manual aiming movements, rather inconsistent results have been reported. For instance, Bradshaw et al. (1988) had right-handed participants perform continuous reciprocating (leftto-right and right-to-left) tapping movements with their preferred and non-preferred hands between targets arranged in two columns on a stimulus board (as fast as possible). The stimulus board was placed either centrally, or entirely to the left or right of the body midline, thus requiring the participants to perform movements crossing the body midline, or movements in ipsilateral or contralateral space (depending on which hand was used for responding), respectively. Results showed that there was an adductive superiority, regardless of the position of the stimulus board. In a later study by the same authors (1990), both dextrals and sinistrals as fast as possible performed a similar tapping task with their preferred and non-preferred hand, using a centrally placed stimulus board. Results again revealed an adductive superiority in both dextrals and sinistrals, and for both the preferred and the non-preferred hand. Note, however, that besides an interpretation based on movement type, an alternative interpretation in terms of movement direction is also possible. That is, the Bradshaw et al. $(1988,1990)$ results might also reflect a leftward superiority for the right hand and a rightward superiority for the left hand. Morgan et al. (1994) used a similar task, requiring their participants to perform continuous drawing movements between targets arranged in two columns presented on a graphics tablet using both their preferred (i.e., right) and non-preferred (i.e., left) hand. The graphics tablet was placed before the participants' midline. In contrast to the results presented by Bradshaw et al. (1988, 1990), Morgan et al. (1994) observed that the right hand showed an adductive advantage, whereas the left hand showed an abductive advantage. This observation has been interpreted as evidence that right-handers have a propensity for right-to-left over left-to-right movements.

Regarding discrete aiming responses, the findings are in general straightforward, although often different terms are used for the interpretation of these findings. For example, Carey et al. (1996) had right-handed participants perform right- and left-hand pointing and anti-pointing movements towards targets located either on the left or the right of a central start position. Anti-pointing refers to pointing movements to the mirror-symmetrical position (i.e., the opposite side of the central fixation point) from the presented stimulus position. They found that for both pointing and anti-pointing movements, movements in ipsilateral space were performed in significantly shorter time periods compared to those in contralateral space. Note that, given the definitions provided earlier in this introduction, the results of the Carey et al. (1996) study might also be interpreted in terms of an abductive superiority. Alternately, the results might reflect a right hand propensity for rightward movements, but a left hand propensity for leftward movements. In the aforementioned study by Bradshaw et al. (1990), right-handed participants were also required to perform discrete aiming movements toward a single target (located on the left or the right of the starting position). Movements were either adductive or abductive in nature, were performed within ipsilateral space (no crossing of the body midline), and were to be executed as fast as possible. Results now revealed an abductive superiority for both the preferred and the non-preferred hand (which can also be classified as a rightward superiority for the right hand and a leftward superiority for the left hand). Thus, despite of the similarity in the findings of the Carey et al. (1996) and Bradshaw et al. (1990) studies, there is a great deal of inconsistency in the terms used to describe these findings.

The aforementioned examples of studies on discrete manual aiming involved aiming movements to a target presented in isolation. However, there have been studies on discrete manual aiming movements in which, besides a target, also an irrelevant distracting stimulus is presented. Tipper et al. (1992) required participants to quickly and accurately reach for and depress a button indicated by a red target light, leaving from a central start position. On some trials, the target light was accompanied by a yellow distractor light that was presented simultaneously elsewhere on the display. Participants were instructed to ignore this distracting information. Tipper et al. (1992) found that the presence of such a distractor prolonged the time needed to respond to the target stimulus (i.e., distractor interference). Moreover, regardless of which hand was used for responding, they observed that, when reaching for a target that was presented at the midsagittal plane, distractors on the ipsilateral side of space caused more interference than did distractors on the contralateral side of space. This phenomenon has been called the ipsilateral effect. Meegan and Tipper (1998, 1999) replicated this finding and proposed the visuomotor processing hypothesis to account for these findings. According to this hypothesis, distractor interference in manual aiming is attributable to visuomotor competition from distractors; distractor interference reflects the need (and the time it takes) to suppress or inhibit responses toward the distractor. The visuomotor processing hypothesis further suggests that the faster a response can be made toward a particular 
location, the more interference a distractor at that location will produce (Meegan and Tipper, 1999). The idea here is that the identifying features of objects (i.e., visual information for distinguishing target and distractor: vision-for-identification) and the visuomotor features of objects (i.e., visual information for the control of actions afforded by that object: vision-for-action) are processed in parallel by the visual system. Vision-foridentification, however, is relatively slow compared to vision-for-action, as a result of which the visuomotor representation of the distractor can be at an advanced level when information comes available for selection of the desired target. And the greater the advancement of the visuomotor representation of the distractor, the more time it takes to inhibit a response toward the distractor. Thus, the faster a reaching movement can be made to a location, the greater the advancement of the visuomotor representation of a distractor appearing at that location (when information comes available for selection), and the greater the interference caused by that distractor.

We have already discussed the visuomotor processing advantages for ipsilateral relative to contralateral locations, so the visuomotor processing hypothesis can account for the ipsilateral effect as reported by Tipper and colleagues (Tipper et al., 1992; Meegan and Tipper, 1998, 1999). However, as was the case in the Carey et al. (1996) study, in the Tipper et al. (1992) and Meegan and Tipper (1999) studies hemispace (i.e., ipsilateral vs. contralateral) was confounded with movement direction in terms of rightward and leftward, or with movement type in terms of abduction and adduction. After all, ipsilateral locations were associated with rightward movements when responding with the right hand, but leftward movements when responding with the left hand. Thus, the finding of distractor interference being more pronounced for ipsilateral compared to contralateral distractors might also be interpreted in terms of right-hand responses being more susceptible to interference from distractors affording rightward movements, and left-hand responses being more susceptible to interference from distractors affording leftward movements. In the light of the visuomotor processing hypothesis this would require that the right hand is superior at performing left-to-right rather than right-to-left movements, whereas the opposite is true for left-hand movements. Alternately, the Tipper et al. (1992) and Meegan and Tipper (1999) results might also be interpreted in terms of more interference from distractors affording abductive movements compared to distractors affording adductive movements. This would then require that in discrete target-directed aiming movements there is an advantage for abductive over adductive movements.

To sum up, it appears that the findings on discrete manual aiming performance are rather consistent, but in a way also confounded. That is, it remains unclear whether the observed advantages for movements towards particular spatial locations (and the stronger interference caused by distractors at those locations) are associated with an ipsilateral advantage (Carey et al., 1996), an abductive advantage (Bradshaw et al., 1988, 1990), or a rightward advantage for right-hand movements and a leftward advantage for left-hand movements. In the present study we sought to tear apart the respective effects of hemispace, movement direction, and movement type on manual aiming performance in the presence of distracting stimuli.

In the first experiment, right-handed participants performed rightward and leftward movements with their preferred hand within the left or the right hemispace. In that way, we were able to distinguish between the effect of movement direction (rightward vs. leftward movements) and hemispace (ipsilateral $v s$. contralateral movements) on distractor interference. In the second experiment, a new group of right-handers participated in the same experiment, but now they performed with both their preferred (right) and non-preferred (left) hand, thus enabling us to unconfound movement direction in terms of rightward and leftward movements ánd movement type in terms of abductions and adductions.

In both Experiments 1 and 2, participants were instructed to move the eyes to the target box as soon as the index finger of the responding hand had contacted the start location. This was prompted and facilitated by blinking the target box three times during a period of $1000 \mathrm{msec}$. The rationale for this manipulation was to reduce the effect of the use of differential eye fixation strategies when performing the present task. That is, without applying the present manipulation some participants may have their eyes directed at the start position at stimulus onset, whereas others might adopt a strategy of fixating the position where the target is to appear. In the former case, target and distractor will be presented in peripheral vision, whereas in the latter target and distractor will be presented foveally. Because in the present study discrimination of target and distractor is based on color, participants adopting the latter strategy would have an advantage, as color is processed in foveal vision. We therefore chose to bring eye position at target onset under experimental control.

\section{EXPERIMENT 1}

\section{Method}

\section{Participants}

Fourteen Maastricht University undergraduate students, 7 female and 7 male, participated. Mean age was 22.6 years (range 20-25). In this, and the 
next experiment, they were paid the equivalent of about 5 US\$, were right-handed (as indicated by self-report), had normal or corrected-to-normal visual acuity, and were naive as to the purposes of the experiment.

\section{Materials and Apparatus}

Participants were tested individually in a quiet, dimly illuminated room. They were seated on a height-adjustable chair in front of a $43.2 \mathrm{~cm}$ (i.e., 17 inch) video monitor that was equipped with a touch screen. The monitor was placed in a normal, upright position and participants made responses with the tip of the right index finger directly on the screen. The stimulus display was made up of a start box and a target box, both in white outline on a black background. The target box was always located in the center of the screen. The start box was located either to the left or to the right of the target box, at a distance of $12 \mathrm{~cm}$ (side-to-side). Participants were positioned such that the body midline was in line either with the start box or with the target box, and the shoulder line (i.e., an imaginary line connecting both shoulders) was parallel to the monitor's screen. The stimulus displays used in the experiment and the position of the participant's body midline relative to the start position and the target are schematically depicted in Figure 1. The target stimulus was presented as a green square completely filling the target box. The distractor stimulus was presented as a red square appearing either in between the start box and the target or beyond the target. The distractor could appear at one of four possible target-distractor distances (side-to-side): 2, 14, 26, and $38 \mathrm{~mm}$. Start box, target box, and distractor box were all $10 \mathrm{~mm}$ wide and high. Note, however, that for defining an aiming error the effective target width was set at $12 \mathrm{~mm}$; this was done in order to limit the number of errors (i.e., target misses).

\section{Design}

Participants performed in two sessions, each lasting about 20 minutes. There were four experimental conditions (i.e., two in each session), resulting from the orthogonal combination of movement direction (rightward or leftward) and hemispace in which the movement was performed (right or left). Thus, there were four experimental conditions: (1) rightward, right hemispace; (2) rightward, left hemispace; (3) leftward, right hemispace; and (4) leftward, left hemispace. Rightward refers to movements starting from the left start position and moving to the right. Leftward refers to movements starting from the right start position and moving to the left. Right hemispace indicates that the movement is performed within the side of space right from the body midline. Left hemispace indicates that the movement is

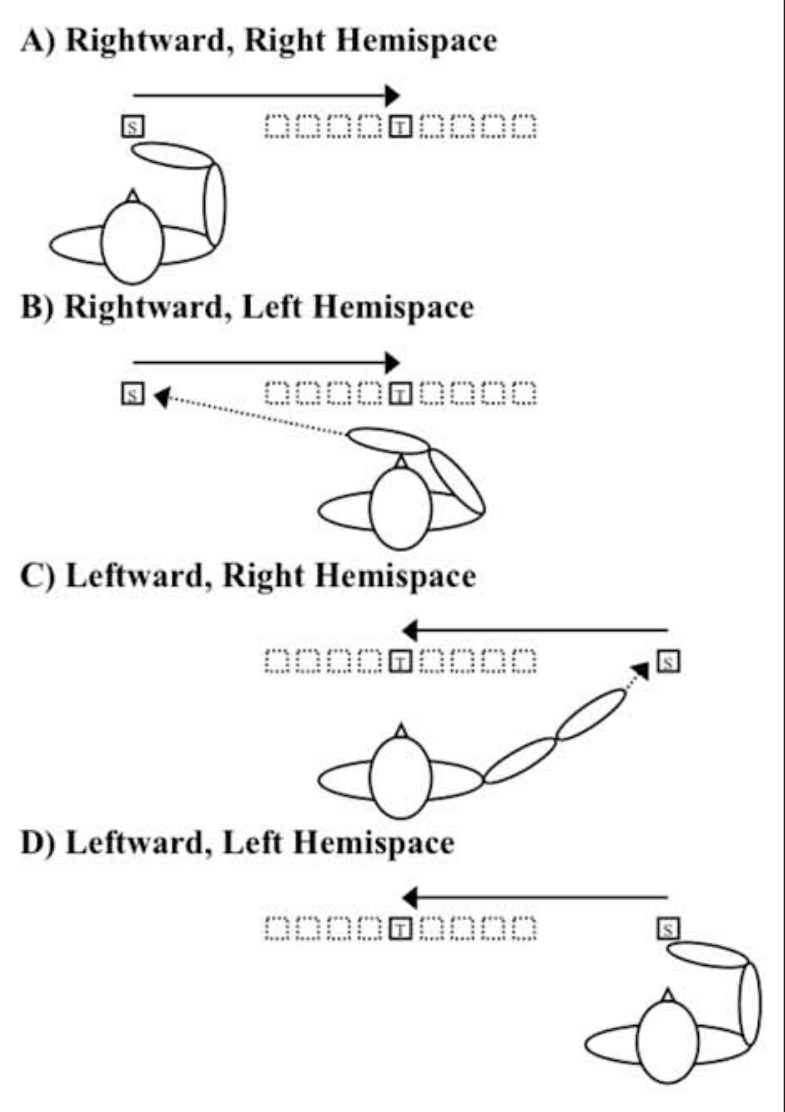

Fig. 1 - Schematic depiction of the stimulus displays used in the present study, and the position of the participant relative to the start position and the target. The " $S$ " indicates the start position of the responding hand. The " $T$ " indicates the location of the target. The dotted squares on either side of the target indicate the possible locations of the distractor stimulus. The dotted arrow leaving from the arm of the geometric participant indicates the position the participant's hand departs from. The solid arrow indicates the movement direction. The " $S$ ", " $T$ ", and the arrows were not actually present.

performed within the side of space left from the body midline. For each experimental condition there were 144 test trials, preceded by 25 practice trials. Within a block of 144 test trials, there were 24 trials without distractor $(=16.7 \%)$ and 120 with distractor $(=83.3 \%)$. For each of the 4 possible distractor locations at both sides there were 15 trials. Order of distractor trials and no-distractor trials was random. Order of experimental conditions was random.

\section{Procedure}

At the beginning of each trial, the start box turned green, indicating that the participant could move the fingertip to the green start box. Participants were informed that on each trial a green light would appear in the target box and that on some trials a red light would appear in a different location. Participants were asked to first contact the start box with the index fingertip of the right (i.e., preferred) hand and then to move the eyes to the target box. The shift in gaze position 
was prompted and facilitated by blinking the target box three times during a period of $1000 \mathrm{msec}$. Then, after an additional delay of $1000 \mathrm{msec}$, the target stimulus (with or without distractor) was presented. Participants were instructed to move to and contact the green target box as quickly and accurately as possible while ignoring the red distractor box. If they missed the target, they were encouraged to try to do better in subsequent trials. One second after completing the aiming movement, the start box turned green again, signaling the start of the next trial which the participants could initiate at will. The computer presented a visual feedback signal if the participant failed to hit the target box or if the start box was released too soon (that is within $150 \mathrm{msec}$ after target presentation).

We calculated two measures of response time: (1) reaction time (RT), measured from the time when the target stimulus appeared to the time when the start box was released; and (2) movement time (MT), measured from the time when the start box was released to when the target box was contacted.

\section{Analysis}

RTs below 150 msec were considered anticipations and were excluded from data analyses. RTs over $1000 \mathrm{msec}$ as well as MTs below $150 \mathrm{msec}$ or over $1000 \mathrm{msec}$ were considered outliers, and were also excluded; $15.0 \%$ of the trials were removed using these criteria (14.1\% in the no-distractor and $16.0 \%$ in distractor condition). This was mostly due to anticipations (14.8\%). RT, MT, and percentage errors were calculated for each subject as a function of movement direction, hemispace, and distractor presence. Analysis of variance (ANOVA) was performed on mean RT, MT, and percentage errors with movement direction (rightward vs. leftward), hemispace (right vs. left), and distractor presence (without vs. with distractor) as within-subject variables. Error trials (i.e., trials on which the participant failed to hit the target) were excluded from all latency analyses ${ }^{1}$.

Whenever needed, the tests were adjusted for heterogeneity of variance and covariances using the Huynh-Feldt corrected significance values. Posthoc analyses were carried out using Tukey' $\mathrm{s}$ honestly significant difference (HSD) procedure; an alpha level of .05 was employed to determine statistical significance.

\footnotetext{
${ }^{1}$ Although it was not the focus of the present study, we also analyzed the effect of target-distractor separation $(8 v s .32 \mathrm{~mm}$; we averaged the 2 and $14 \mathrm{~mm}$ and the 26 and $38 \mathrm{~mm}$ distances to constrain the degree of variability) and distractor location (in between start and target $v s$. beyond the target) on MT Interference and movement endpoint, the latter in terms of constant error (ce). There was more distractor interference in MT with the small target-distractor separation than with the large target-distractor separation. Furthermore, movement endpoints were biased away from the location of the distractor. This bias was larger for the small target-distractor separation than for the large target-distractor separation. The observed effects were similar to our previous findings (Keulen et al., 2002, 2003).
}

\section{Results}

\section{Reaction Time}

There was a significant distractor presence main effect, $\mathrm{F}(1,13)=37.427, \mathrm{p}<.001$, indicating that RTs were longer for trials without distractor compared to trials with distractor (mean $=272$ and $242 \mathrm{msec}$, respectively, for trials without and with distractor). All remaining F-values involving movement direction, hemispace, and distractor presence were non-significant (all ps > .1).

\section{Movement Time}

There was a significant movement direction main effect, $\mathrm{F}(1,13)=41.507, \mathrm{p}<.001$, indicating that leftward movements were shorter in duration than rightward movements (mean $=324$ and $385 \mathrm{msec}$, respectively). The hemispace main effect was also significant, $F(1,13)=13.035, \mathrm{p}<$ .005 , indicating that movements performed within the right hemispace were shorter in duration compared to movements performed within the left hemispace (mean $=339$ vs. 370 msec, respectively). The significant distractor presence main effect, $\mathrm{F}(1,13)=27.592, \mathrm{p}<.001$, indicated that MTs were significantly longer in the distractor condition compared to the no-distractor condition (mean $=358 v s .351 \mathrm{msec}$, for trials with and without distractor, respectively).

Importantly, the distractor presence main effect was qualified by a significant distractor presence $x$ movement direction interaction, $\mathrm{F}(1,13)=8.135$, $\mathrm{p}<.05$, indicating less interference for leftward movements compared to rightward movements (i.e., 3 vs. $11 \mathrm{msec}$, respectively; see Figure 2). Note, that the $3 \mathrm{msec}$ interference effect observed with the leftward movements barely missed significance $(p=.075)$. All remaining F-values involving movement direction, hemispace, and distractor presence were non-significant.

\section{Percentage Errors}

All F-values involving movement direction, hemispace, and distractor presence were nonsignificant. Mean error percentage was $5.6 \%$.

\section{Discussion}

In the present experiment we investigated the effect of movement direction and hemispace on the amount of interference caused by a distractor stimulus. Right-handed participants performed right-hand movements to the right or to the left, either within the right or the left hemispace. Results showed that leftward movements were shorter in duration than rightward movements, and that movements performed within the right hemispace (i.e., ipsilateral movements) were 


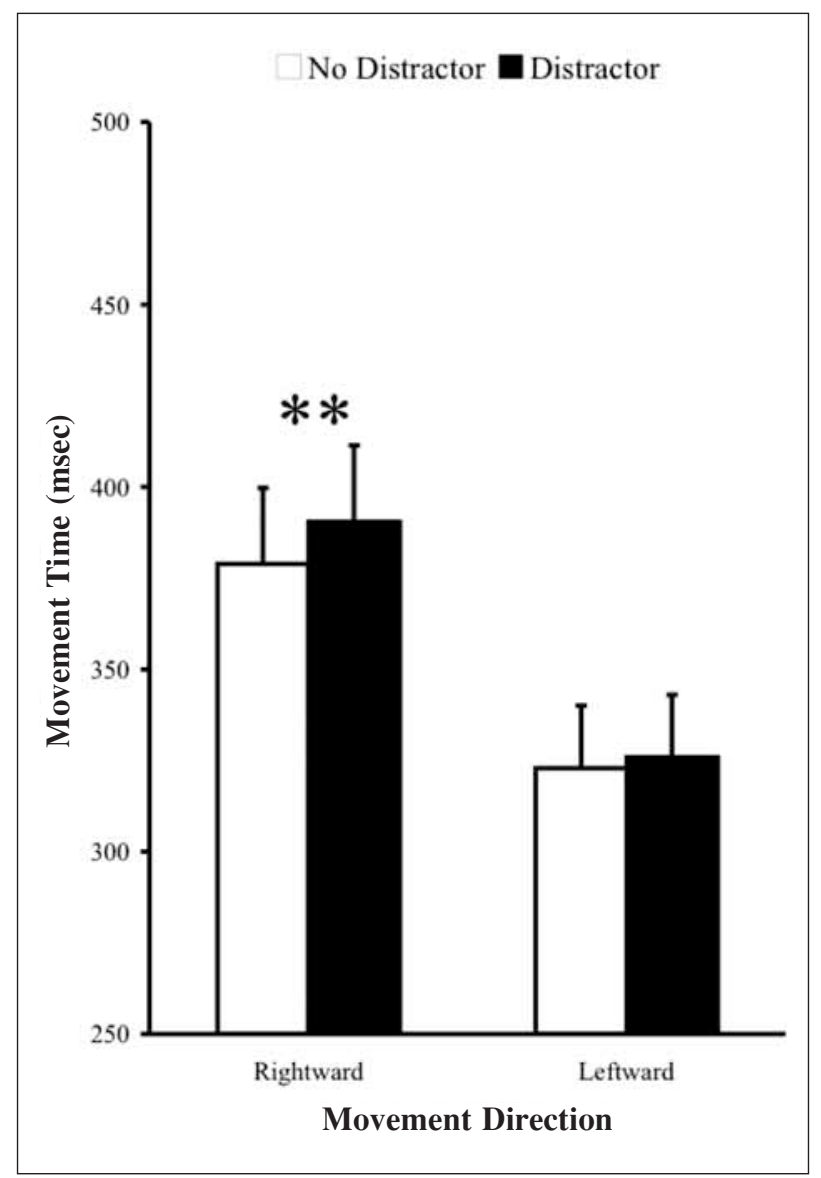

Fig. 2 - Movement time as a function of movement direction and distractor presence in Experiment 1. The error bars represent standard errors; ** indicates significance at the .005 level.

shorter in duration than movements performed within the left hemispace (i.e., contralateral movements). Distractor interference occurred in MT and, importantly, this interference effect was larger in rightward movements than in leftward movements. Finally, RTs were shortened by the presence of a distractor.

We found less interference for leftward compared to rightward movements performed with the right hand. This finding is incompatible with the visuomotor processing hypothesis, as this would imply that the observed superiority for leftward movements performed with the right hand would consequently result in more interference from distractors affording leftward movements, which was not the case. Thus, the hypothesized relationship between directional superiority and distraction susceptibility did not hold in the present selective reaching task. In the general discussion of this paper we will offer a possible explanation for this discrepancy.

Although we observed distraction susceptibility to be more pronounced for rightward compared to leftward responses, it remains ambiguous whether movement direction is in fact the driving factor or movement type, because these two factors were confounded. That is, whereas rightward movements were abductive, leftward movements were adductive. As a result, the reduced amount of interference observed for leftward movements compared to rightward movements might either be a "movement direction" effect or a "movement type" effect. Assuming a movement direction effect, this would imply that leftward movements are less prone to distractor interference than rightward movements. A movement type effect, on the other hand, would imply that adduction movements are less prone to distractor interference than abduction movements. To differentiate between a "movement direction" account and a "movement type" account, we performed a second experiment in which participants performed leftward and rightward movements with both their right and left hands. If movement direction is responsible for the difference in interference, then leftward movements should show less interference than rightward movements, irrespective of the hand used to respond. However, if movement type is the crucial variable, then a dissociation between righthand and left-hand movements should occur. That is, with the right hand there should be less interference for leftward (adductive) than for rightward (abductive) movements. In contrast, with the left hand there should be less interference for rightward (adductive) than for leftward (abductive) movements.

\section{EXPERIMENT 2}

\section{Method}

\section{Participants}

Fourteen new right-handed students, 8 female and 6 male, participated. Mean age was 23.1 years (range 20-27).

\section{Materials and Apparatus}

The same experimental setup as in Experiment 1 was used. The only difference was that participants were positioned such that the body midline was always in line with the target box, and that they made movements with both their right and their left hand (see Figure $1 \mathrm{~B}$ and $\mathrm{C}$ ).

\section{Design}

Participants again performed in two sessions, each lasting about 20 minutes. There were four experimental conditions (i.e., two in each session), resulting from the orthogonal combination of movement direction (rightward or leftward) and hand used for responding (right or left). The four conditions were: (1) right-hand rightward; (2) righthand leftward; (3) left-hand rightward; and (4) lefthand leftward. Rightward refers to movements 
starting from the left starting position. Leftward refers to movements starting from the right starting position. In one session participants performed either the two right-hand conditions or the two lefthand conditions. Order of the conditions within one session was random. The number of trials in each condition was identical to that in Experiment 1.

\section{Procedure}

Procedure was identical to the procedure in Experiment 1.

\section{Analysis}

Using the Experiment 1 criteria for exclusion of trials resulted in $10.3 \%$ of the trials being removed (9.1\% in the no-distractor and $11.5 \%$ in distractor condition). Again, the majority of excluded trials was due to anticipations $(8.5 \%)$. RT, MT, and Percentage errors were calculated for each subject as a function of hand, movement direction, and distractor presence. We performed ANOVA on mean RT, MT, and percentage errors with hand (right $v s$. left), movement direction (rightward $v s$. leftward), and distractor presence (without $v s$. with distractor) as within-subject variables ${ }^{2}$.

\section{Results}

\section{Reaction Time}

There was a significant distractor presence main effect, $\mathrm{F}(1,13)=169.335, \mathrm{p}<.001$, indicating that RTs were longer for trials without distractor compared to trials with distractor $($ mean $=264$ and $236 \mathrm{msec}$, respectively, for trials without and with distractor). All remaining F-values involving hand, movement direction, and distractor presence were non-significant (all ps > .05).

\section{Movement Time}

There was a significant hand main effect, F (1, $13)=7.694, p<.05$, indicating that movements performed with the right hand were shorter in duration than movements performed with the left hand (mean = 401 and $432 \mathrm{msec}$, respectively). The hand main effect was qualified by a significant hand $\times$ movement direction interaction, $F(1,13)=$ $54.351, \mathrm{p}<.001$, indicating that the right hand was better at executing leftward movements, whereas the left hand was better at executing rightward movements. There was a significant distractor

\footnotetext{
${ }^{2}$ Analysis of the effect of target-distractor separation $(8 v s .32 \mathrm{~mm})$ and distractor location (in between start and target $v s$. beyond the target) on MT Interference and movement endpoint again revealed more distractor interference in MT with the small target-distractor separation than with the large target-distractor separation. Furthermore, movement endpoints were biased away from the location of the distractor. This bias was larger for the small target-distractor separation than for the large target-distractor separation.
}

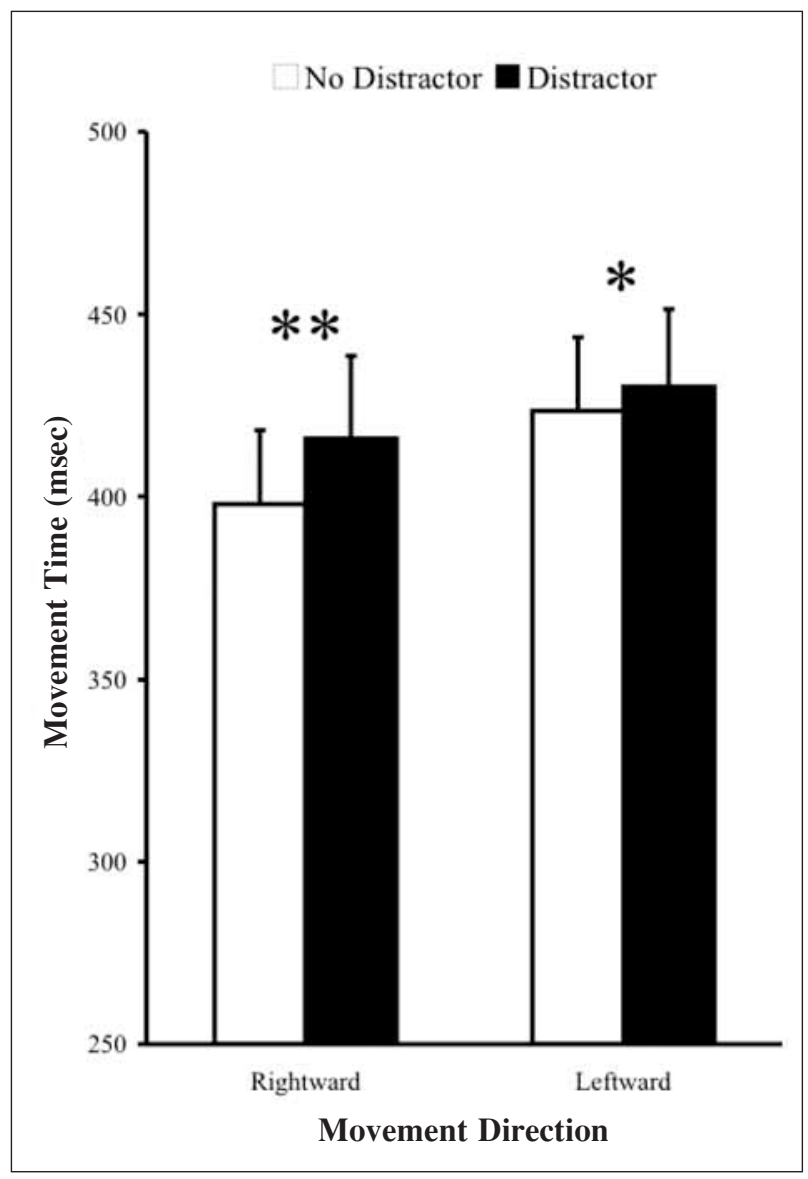

Fig. 3 - Movement time as a function of movement direction and distractor presence in Experiment 2. The error bars represent standard errors; * indicates significance at the .05 level; ** indicates significance at the .005 level.

presence main effect, $\mathrm{F}(1,13)=14.813, \mathrm{p}<.005$. That is, MTs were significantly longer in the distractor condition compared to the no-distractor condition (mean $=423 \mathrm{vs}$. $411 \mathrm{msec}$, for trials with and without distractor, respectively).

Importantly, the distractor presence main effect was qualified by a significant movement direction $\times$ distractor presence interaction, $F(1,13)=$ $11.060, \mathrm{p}<.01$. This interaction indicated less interference for leftward movements compared to rightward movements (i.e., 7 vs. $18 \mathrm{msec}$, respectively; see Figure 3). This effect was independent of the hand used for responding, as there was no significant hand $\times$ movement direction $\times$ distractor presence interaction, $\mathrm{F}(1,13)$ $=.501, \mathrm{p}>.4$. All remaining F-values involving hand, movement direction, and distractor presence were non-significant.

\section{Percentage Errors}

The presence of a distractor slightly reduced the percentage errors $[$ mean $=4.3 \mathrm{vs} .3 .3 \%$, for trials without and with distractor, respectively; F (1, 13) $=7.052, \mathrm{p}<.05]$. However, this effect was qualified by a significant distractor presence $\times$ hand interaction, $\mathrm{F}(1,13)=4.990, \mathrm{p}<.05$, 
indicating that it only materialized for movements performed with the right hand (i.e., $4.6 \mathrm{vs}$. 2.8\%, for trials without and with distractor, respectively; $\mathrm{p}<.05$ ), not for movements performed with the left hand (i.e., $4.0 \mathrm{vs} .3 .8 \%$, for trials without and with distractor, respectively; $\mathrm{p}>.6$ ).

\section{Discussion}

Experiment 2 was designed to differentiate between a "movement direction" and a "type of movement" interpretation for explaining the key finding of Experiment 1 that leftward movements suffered less interference from the presence of a distractor than rightward movements. Results again showed that there was less interference for a leftward movement than for a rightward movement and, most importantly, this effect was independent of the hand used to respond. This outcome solves the ambiguity that was evident in Experiment 1 (confounding of movement direction with type of movement), because the present results showed that, regardless of the responding hand, leftward movements were associated with less interference than rightward movements. This observation indicates that movement direction rather than type of movement was responsible for the difference in interference between leftward and rightward movements.

\section{General Discussion}

In the present study we investigated the effects of hemispace, movement direction, and type of movement on distractor interference in a goaldirected reaching task performed by right-handers. The results of two experiments consistently showed that leftward movements suffered less MT interference than rightward movements. We also found that reaction times were shortened by the presence of a distractor. Furthermore, movements were executed faster within ipsilateral space than within contralateral space, and adductions were faster than abductions. Right hand movements were faster than left hand movements. Below these findings will be discussed in separate paragraphs.

\section{Greater Distraction Susceptibility for Leftward over Rightward Movements}

A new and most interesting finding was that leftward movements were less prone to distractor interference than rightward movements, regardless of the hand used for responding and regardless of the hemispace in which the movement was performed. How to explain this most intriguing phenomenon? In our experiments, we made sure that the participants had their eyes on the target location before target (and distractor) were presented, thus assuring that target and distractor were presented foveally rather than peripherally. An additional consequence of this manipulation was that in the present study, the eyes were at the target even before hand movement began, as a result of which leftward responses always took place in the right visual field (which is processed initially by the primary visual cortex of the left hemisphere), whereas rightward responses always took place in the left visual field (which is processed initially by the primary visual cortex of the right hemisphere). Note, that the situation of eye movements preceeding hand movements (although experimentally induced in the present study) is consistent with the so-called "eyes-first, hand-second phenomenon" (e.g., Abrams et al., 1990; Neggers and Bekkering, 2001), suggesting that when pointing to or reaching for a target the eyes arrive at the target well before the hand does (which does not necessarily imply that hand movement does not start until eye movement has been completed). The argument that visual information regarding the ongoing response is projected to different hemispheres, depending on the direction (i.e., rightward or leftward) of the response, allows for an explanation of the observed superiority for leftward movements in terms of a cerebral lateralization and specialization account.

In particular, it has been proposed that in righthanders the left hemisphere is superior at processing (visual and non-visual) feedback information during goal-directed aiming movements (e.g., Flowers, 1975; Todor and Doane, 1978; Roy et al., 1994; Mieschke et al., 2001). This position is based on several lines of evidence. Flowers (1975) asked participants to perform two types of tasks: a visually controlled, discrete aiming task and a ballistic tapping task. Flowers (1975) found that right hand advantages (in righthanders) were more pronounced for aiming than for tapping, and that the hand differences in aiming increased with the accuracy demands of the aiming task. A similar observation was made by Todor and Doane (1978) who reported a right hand advantage that was greater for small than large targets. Furthermore, Roy et al. (1994) reported that in right-handed participants performing aiming movements, the right hand was associated with smaller movement times than the left hand. Kinematic analyses revealed that the right and the left hand differed only in time spent after peak velocity, with the right hand spending significantly less time in deceleration, regardless of the availability of vision. On the basis of this finding, Roy et al. (1994) argued that the left hemisphere may be more efficient at processing (visual or nonvisual) feedback information.

It is a common notion that online feedbackprocessing plays an important role in targetdirected reaching, especially in the presence of nearby distractors. That is, in the later stages of the movement, when the critical task is to terminate 
the movement accurately on the target and not on the distractor, feedback regarding the ongoing movement in relation to the target and distractor is necessary to prevent hitting the distractor instead of hitting the target. When performing target-directed movements within the right visual field, visual information regarding the moving hand is projected directly to the left hemisphere that is superior at processing feedback during the ongoing movement. However, when performing target-directed movements in the left-visual field, visual information is projected to the right hemisphere, and a small, though significant time-consuming process of interhemispheric communication is needed to consult the specialized, dominant left hemisphere. According to this account, the superiority of leftward over rightward movements in solving distractor interference is related to a left hemisphere superiority in online feedbackprocessing during goal-directed movements in right-handers.

\section{Distractor Facilitation, not Interference, in Reaction Times}

The observation that distractor interference occurred in movement time, and that reaction times were facilitated by (rather than that they suffered from the presence of) the distractor is in line with some of our previous studies (Keulen et al., 2002, 2003) reporting a similar outcome. In those studies we suggested that this result might have been caused by the fact that movement direction (and, in the present study also target location) was known in advance throughout the experiment. So it might be the case that RTs mainly reflected detection of the stimulus (i.e., target alone or target plus distractor) and initiation of the response. Detectability of the onset of the target, however, might have been easier when the target was accompanied by a distractor than when the target was presented alone. This is because total stimulus energy is larger when target and distractor are presented together than when the target is presented alone (e.g., Nickerson, 1973).

\section{Ipsilateral Advantage in Target-Directed Aiming Movements}

The shorter movement latency of ipsilateral compared to contralateral movements is a rather common observation. Several explanations have been forwarded to account for this ipsilateral movement advantage. One possible explanation is concerned with biomechanical differences between ipsilateral and contralateral movements. Carey and colleagues (Carey et al., 1996; Carey and Otto-de Haart, 2001) argued that factors such as a larger displacement of the center of mass of the limb and more muscle groups to be recruited might be responsible for the longer latencies of movements crossing the midsagittal plane. Another explanation focuses on interhemispheric processes. More specifically, when the visual input is given to one hemisphere and the motor response is mediated by the other (which is the case with contralateral targets), interhemispheric transfer of information is required. Within-hemisphere processing of feedback information arising from the reaching limb is carried out more efficiently than crosshemisphere processing, so this difference might account for the ipsilateral movement advantage (Velay and Benoit-Dubrocard, 1999; Velay et al., 2002). However, in the present study the eyes were directed at the target location at stimulus onset, so the target stimulus was actually presented centrally rather than ipsilaterally or contralaterally. Thus, the explanation based on interhemispheric processes might not be appropriate to explain the present ipsilateral advantage.

\section{Adductive Advantage for Right-Hand and Left-Hand Aiming Movements}

In the present study visual information regarding left-to-right hand movements was always presented to the right hemisphere, whereas visual information regarding right-to-left hand movements was always presented to the left hemisphere (regardless of which hand was used for responding). One might therefore expect that both the right and the left hand would be superior at leftward responses, as these responses were performed within the visual field that was attentionally monitored by the left hemisphere (which is superior at processing feedback regarding the ongoing movement). However, we observed that only the right hand demonstrated a leftward advantage; the left hand demonstrated a rightward advantage. A possible explanation for this finding might be as follows. Whereas movement of the right arm/hand system is predominantly controlled by the left hemisphere, movement of the left arm/hand system is predominantly controlled by the right hemisphere. Efficient visually guided movement requires that information regarding the position of the hand is transported to the hemisphere that controls the ongoing movement, so that this information can be used to fine-tune the ongoing movement. When moving leftward with the right hand or rightward with the left hand, visual information regarding the moving hand is projected directly to the hemisphere that controls movement of that particular hand, thus minimizing the need for interhemispheric cross talk. On the other hand, when moving rightward with the right hand or leftward with the left hand, visual information regarding the moving hand is projected to the hemisphere opposite to the hemisphere that controls movement of that particular hand. It is therefore plausible to assume that leftward movements with the right hand and rightward 
movements with the left hand are executed more efficiently (i.e., faster) compared to rightward movements with the right hand and leftward movements with the left hand, respectively, as was the case in the present study.

\section{Manual Asymmetries: Right-Hand Advantage for Movement Execution}

The right-hand advantage for movement execution in right-handers is well documented, and has been attributed to a greater ability of the left hemisphere for the processing of perceptual and/or motor information during the ongoing movement (Flowers, 1975). Because reaches toward small targets are assumed to rely heavily on feedback, they are performed best by the arm that is linked to the left hemisphere (i.e., the right arm). This suggestion is supported by the finding by Todor and Doane (1978) that the right-hand advantage for MT is dependent on the size of the target, with the advantage being larger for small targets. Another explanation might be that the right (i.e., preferred) hand benefits from an overpractice in aiming tasks. This overpractice results in differences in the specification and timing of the muscular forces required to move the limb towards the target (Roy and Elliott, 1989).

\section{Directional Superiority vs. Distraction Susceptibility}

In the present study we did not find a relationship between directional superiority and distraction susceptibility, which does not fit with some of the findings reported by Tipper et al. (1992). That is, they found that distractors in locations which have a visuomotor processing advantage (as is reflected in the response times to targets in those particular locations) show larger amounts of distractor interference. We observed that for both the right and the left hand distraction susceptibility was greater for rightward movements, whereas the right hand showed a leftward superiority and the left hand a rightward superiority. Procedural differences between our study and the Tipper et al. (1992) study might have been responsible for this inconsistency. That is, in our study both target and distractor afforded movements in the same direction; there were only distractors affording leftward movements when the required target-directed movement was right-to-left, and there were only distractors affording rightward movements when the required target-directed movement was left-to-right. The relationship between directional superiority and distraction susceptibility reported by Tipper et al. (1992) was observed when the target required a central movement, and the distractor afforded an either ipsilateral or contralateral movement, as a result of which the distractor had either a visuomotor processing advantage or disadvantage relative to the target. In our study the distractor never had a visuomotor processing advantage (or disadvantage) relative to the target due to directional superiority, as target and distractor were always associated with the same movement direction (either rightward or leftward). This might explain the lack of the directional superiority-distraction susceptibility relationship.

\section{Distractor Interference as a Function of Distractor Location}

In the present study we chose not to include distractor location in our primary analysis. One might object to this approach by stating that in the light of a left hemisphere superiority for feedback processing it would be especially interesting to analyze the respective effects of distractors to the left and to the right of the target, as visual information regarding these distractors is projected to different hemispheres. That is, in the present study the eyes were directed at the target location at stimulus onset, as a result of which visual information regarding right distractors reached the left hemisphere, whereas information regarding left distractors reached the right hemisphere. One might thus expect right distractors to cause more interference than left distractors, as the former are projected to the hemisphere that is superior at processing feedback (i.e., the left hemisphere), and therefore have a visuomotor processing advantage (i.e., no need for time-consuming interhemispheric cross talk) relative to left distractors. However, this differential effect is likely to be modified (i.e., counteracted or augmented) by the concurrent operation of alternative effects associated with the location of the distractor. For one, it has been reported frequently that distractors in between the start and the target interfere more than distractors located beyond the target (i.e., proximity-to-hand effects; e.g., Tipper et al., 1992). This would imply that for left-to-right movements there is more interference from left distractors, whereas the opposite is true for right-to-left movements. Another potentially influencing account relates to the contralateral control of aiming movements in combination with the visuomotor processing hypothesis mentioned in the introduction of this paper. It is to be expected that distractors that are processed by the hemisphere that also controls the arm movement will interfere most with the targetdirected aiming movements than those processed by the opposite hemisphere, as the former have a visuomotor processing advantage due to the lack of need for interhemispheric communication. Movements of the right arm are predominantly controlled by the left hemisphere, whereas left arm movements are controlled by the right hemisphere. This implies that for right arm movements right distractors are expected to interfere most with the 
required response, whereas for left arm movements the opposite is true. And finally, when making rightward movements, visual information about left distractors is projected to the same (right) hemisphere as the information about the moving hand, whereas visual information about right distractors is projected to the opposite hemisphere. On the other hand, when making leftward movements, visual information about right distractors is projected to the same (left) hemisphere as the information about the moving hand, whereas visual information about left distractors is projected to the opposite hemisphere. Thus, when moving to the right, there is a visuomotor processing advantage for left distractors, whereas the opposite is true when moving to the left. In other words, in case of rightward movements left distractors will produce more interference compared to right distractors, whereas the opposite is true for leftward movements. The possible concurrent operation of these differential effects hampers the predictions regarding the effects of distractor location on the amount of interference caused by these distractors, because the relative contribution of the various effects to the overall distractor location effect is difficult to predict.

\section{CONCLUSION}

Two experiments revealed an intriguing new phenomenon, namely that right-handers appear to be better in resisting distractor interference when performing leftward compared to rightward movements (in right and left visual fields, respectively). This phenomenon was interpreted in terms of a left hemisphere superiority in feedbackprocessing during online control of goal-directed reaching movements.

\section{REFERENCES}

Abrams RA, Meyer DE and Kornblum S. Eye-hand coordination: Oculomotor control in rapid aimed limb movements. Journal of Experimental Psychology: Human Perception and Performance, 16: 248-267, 1990.

Bradshaw JL, Bradshaw JA and Nettleton NC. Movement initiation and control: Abduction, adduction and locus of limb. Neuropsychologia, 26: 701-709, 1988.
Bradshaw JL, Bradshaw JA and Nettleton NC. Abduction, adduction and hand differences in simple and serial movements. Neuropsychologia, 28: 917-931, 1990.

Carey DP, Hargreaves EL and Goodale MA. Reaching to ipsilateral or contralateral targets: Within-hemisphere visuomotor processing cannot explain hemispatial differences in motor control. Experimental Brain Research, 112: 496-504, 1996.

CARey DP and Otto-De HaART EG. Hemispatial differences in visually guided aiming are neither hemispatial nor visual. Neuropsychologia, 39: 885-894, 2001.

Flowers K. Handedness and controlled movement. British Journal of Psychology, 66: 39-52, 1975.

Keulen RF, Adam JJ, Fischer MH, Kuipers H and Jolles J. Selective reaching: Evidence for multiple frames of reference. Journal of Experimental Psychology: Human Perception and Performance, 28: 515-526, 2002.

Keulen RF, AdAm JJ, Fischer MH, Kuipers H and Jolles J. Distractor interference in selective reaching: Dissociating distance and grouping effects. Journal of Motor Behavior, 35 . 119-126, 2003.

MeEgAn DV and TIPPER SP. Reaching into cluttered visual environments: Spatial and temporal influences of distracting objects. Quarterly Journal of Experimental Psychology, 51: 225-249, 1998

Meegan DV and Tipper SP. Visual search and target-directed action. Journal of Experimental Psychology: Human Perception and Performance, 25: 1347-1361, 1999.

Mieschke PE, Elliott D, Helsen WF, Carson RG and Coull JA. Manual asymmetries in the preparation and control of goal-directed movements. Brain and Cognition, 45: 129-140, 2001.

Morgan M, Bradshaw JL, Phillips JG, Mattingley JB, Iansek $\mathrm{R}$ and BRADSHAW JA. Effects of hand and age upon abductive and adductive movements: A kinematic analysis. Brain and Cognition, 25: 194-206, 1994.

NegGers SF and BekKering H. Gaze anchoring to a pointing target is present during the entire pointing movement and is driven by a non-visual signal. Journal of Neurophysiology, 86: 961-970, 2001.

NICKERSON RS. Intersensory facilitation of reaction time: Energy summation or preparation enhancement? Psychological Review, 80: 489-509, 1973.

Roy EA and ElLIOT D. Manual asymmetries in aimed movements. Quarterly Journal of Experimental Psychology A, 41: 501-516 1989.

Roy EA, Kalbfleisch L and Elliott D. Kinematic analyses of manual asymmetries in visual aiming movements. Brain and Cognition, 24: 289-295, 1994.

TIPPER SP, LORTIE C and BAYLIS GC. Selective reaching: Evidence for action-centered attention. Journal of Experimental Psychology: Human Perception and Performance, 18: 891905, 1992.

TODOR JI and DoAne T. Handedness and hemispheric asymmetry in the control of movements. Journal of Motor Behavior, 10: 295-300, 1978.

Velay JL and BenOIT-Dubrocard S. Hemispheric asymmetry and interhemispheric transfer in reaching programming. Neuropsychologia, 37: 895-903, 1999.

Velay JL, DafFaure V, GiRaud K and HabiB M. Interhemispheric sensorimotor integration in pointing movements: A study on dyslexic adults. Neuropsychologia, 40: 827-834, 2002.

Jos J. Adam, Department of Movement Sciences Maastricht University, PO Box 616 6200 MD, Maastricht, The Netherlands.

(Received 24 March 2004; reviewed 19 July 2004; revised 4 February 2005; accepted 26 July 2005; Action Editor David Carey) 